\title{
Fusarium oxysporum Silver Nanoparticles; their Characterization and Larvicidal Activity against Aedes Mosquitoes
}

\author{
Nyla Shafqat Sumera ${ }^{1,2 *}$,Sadia Sagar Iqbal ${ }^{2}$, Samrah Tahir Khan ${ }^{3}$, Zia ul Rehman ${ }^{3}$ and Wasim Shehzad ${ }^{4}$ \\ ${ }^{I}$ Department of Physics, Government Associate College for Women, Mustafabad, Lahore 54000, Pakistan \\ ${ }^{2}$ Department of Physics, University of Lahore, 54000, Pakistan \\ ${ }^{3}$ Department of Parasitology, University of Veterinary and Animal Sciences, Lahore, 54000, Pakistan \\ ${ }^{4}$ Institute of Biochemistry and Biotechnology, University of Veterinary and Animal Sciences, Lahore, 54000, Pakistan \\ *For correspondence: nyla_imran@yahoo.co.uk \\ Received 10 December 2020; Accepted 23 April 2021; Published 10 June 2021
}

\begin{abstract}
Fusarium oxysporum is an entomopathogenic fungus, and it has anti-biological activity against larvae of mosquitoes. Aedes mosquitoes are responsible for transmitting different diseases in humans. The use of chemical insecticides against mosquitoes is not eco-friendly option and results in the development of insecticidal resistance in mosquitoes. We investigated a biological control activity against these mosquitoes. In the present study, we cultured a local isolate of $F$. oxysporum from soil samples collected from Lahore, Pakistan and were initially identified based on its morphology and then confirmed through PCR. A product of $339 \mathrm{bp}$ was amplified from the ITS (Internal Transcribed Spacer) gene of the fungus and sequenced afterwards. The sequence was in clad with Fusarium, which was isolated from a mosquito's body in the phylogenetic analysis. Local $F$. oxysporum was cultured and silver NPs (nanoparticles) were prepared. UV-Vis analysis depicted a broad peak at $420 \mathrm{~nm}$ wavelength and a narrow height at $310 \mathrm{~nm}$. X-ray diffraction patterns of NPs indicated the existence of sharp diffraction peaks at $2 \theta$ angles of $32.19^{\circ}, 45.55^{\circ}$ and $64.27^{\circ}$ that can be indexed to the (101), (200) and (220) facets of silver, which agree with the values reported for fcc lattice of silver NPs in International Center for Diffraction Data (ICDD). The SEM (scanning electron microscope) micrograph showed well-defined spherical NPs, which were smooth, isotropic, poly-dispersed, and ranging from $10 \mathrm{~nm}$ to $200 \mathrm{~nm}$. The Zeta potential (ZP) measurements and poly-disparity index of 0.16 by DLS revealed a low variability of particle size and exhibited good physiochemical stability of biosynthesized AgNPs. In the Fourier-transform infrared spectroscopy (FTIR) spectrum of biosynthesized AgNPs, strong bands were analyzed at $3280 \mathrm{~cm}^{-1}$ and $1635 \mathrm{~cm}^{-1} . F$. oxysporum NPs enhanced the anti-biological activity by killing Aedes larvae $7 \mathrm{~h}$ earlier than $F$. oxysporum without NPs. Biological control using entomopathogenic fungi can be the best alternative of the chemical method to control the mosquito population. (C) 2021 Friends Science Publishers
\end{abstract}

Keywords: Fusarium oxysporum; Entomopathogenic fungus; Silver nanoparticles; Fungal nanoparticles; Aedes mosquitoes

\section{Introduction}

Nanoparticles (NPs) generally contain 20-15000 atoms and are considered fundamental molecular building blocks for nanotechnology (Zhao et al. 2014). NP synthesis and its potential exploration to use in various applications in optics, electronics and biomedical sciences are of great scientific interest (Colvin et al. 1994; Becker 1999; Crabtree et al. 2003). NPs possess the unique properties of optical, chemical, magnetic as well as mechanical nature (Khan et al. 2019). Compared to the large particles, these particles have a relatively high fraction of atoms and a wide surface area to volume ratio (Tang and Zheng 2018). The NP serves as a link or bridge between the bulk materials and the molecular and atomic structures (Chakraborty and Pradeep 2017). The nano-technology overlaps different disciplines, so it is easy to rebuild the novel experimental protocols in the synthesis of NPs which are safe, reliable and ecofriendly (Ray 2010).

NPs are majorly categorized into two main types, namely organic and inorganic. Organic NPs include carbon NPs, while, inorganic NPs comprise noble metal NPs (e.g., $\mathrm{Au}$ and $\mathrm{Ag}$ ), semi-conductor NPs $\left(\mathrm{TiO}_{2}\right.$ and $\left.\mathrm{ZnO}_{2}\right)$ and magnetic NPs (Komada 1976; Teixeira et al. 2018; Nagajyothi et al. 2020; Wahid et al. 2020). Because of their adherent functional versatility and material superiority, inorganic NPs are widely used in biological sciences (GinerCasares et al. 2016).

The silver nanoparticles (AgNPs) are the most promising as they show good antibacterial and antimicrobial properties (Zhao and Stevens 1998; Kostadinova et al. 2009). During the last two decades, metal NPs synthesis and

To cite this paper: Sumera NS, SS Iqbal, ST Khan, ZU Rehman, W Shehzad (2021). Fusarium oxysporum silver nanoparticles; their characterization and larvicidal activity against Aedes mosquitoes. Intl J Agric Biol 26:115-124 
its application emerged as a prime research topic in the modern material sciences (Roco 2003). These nano-crystals have been employed in sensitive bio-molecular detection, therapy, diagnostic techniques, anti-microbial and catalysis processes (Wang and Herron 1991; Colvin et al. 1994; Samish et al. 2001; George et al. 2004; Govindarajan et al. 2005). Because of the anti-microbial properties of silver nanoparticles, they are widely used in the medical industry (Crabtree et al. 2003). Silver-impregnated polymers and AgNPs are widely used to prevent bacterial infection in open and burn wounds (Jiang et al. 2004; Rai et al. 2009). Silver embedded fabrics are also used as supporting material in the textile industry (Durán et al. 2007). The earlier synthesis methods involved in the NPs were based on physical and chemical processes. These methods have some shortcomings, such as high-temperature requirement causing more expenditure of energy, need for radiations, employment of toxic chemicals that usually resulted in the liberation of hazardous by-products (Komada 1976; Dolgaev et al. 2002; Kabashin and Meunier 2003; Evanoff and Chumanov 2004; Jiang et al. 2004). These methods also required specialized apparatus. Overcoming these issues, biological systems have proved to be used as an efficient system and a prominent alternative for synthesizing both extracellular and intracellular oriented-NPs (Zhang et al. 2020). The biological systems used include microorganisms, including bacteria, fungi and plants (Mishra et al. 2003; Bar et al. 2009). In comparison to the bacterially synthesized NPs, mycosynthesized AgNPs have several merits. These include tolerance for high metal concentration in the medium, reliable and easy large-scale production, better dissemination of NP (Abdel-Aziz et al. 2017). The amount of protein expressed is much higher than that of a bacterial system (Dyal et al. 2006). The filtration of fungi is conveniently done using a filter press, adopting simple filtration techniques and standard equipment, minimizing the investment and energy consumption over other methods (Devi and Joshi 2015).

Mosquitoes are a group of insects that are notorious for causing a wide range of viral and parasitic diseases (Mbanzulu et al. 2020). It has already been reported that mosquitoes like Aedes spp. (Qureshi et al. 2017) and Anopheles spp. (Rana et al. 2014) are more frequent in densely populated and urbanized areas of Pakistan (Mubbashir et al. 2018). They serve as a vector for viruses such as dengue fever, yellow fever, and chikungunya, as well as parasites of malaria and filariasis (Fradin and Day 2002; Subramaniam et al. 2012; Simonsen and Mwakitalu 2013). The control of mosquitoes is mainly done through chemical insecticides (Ranson et al. 2010). The continuous and injudicious use of these chemicals has led to drug resistance development (Liu 2015; McNair 2015), making the chemical less effective for mosquito control (Nauen 2007). This irrational use of insecticides may lead to an uncontrolled mosquito population. It also causes bioaccumulation, unbalancing the ecology as inducing bio- magnification in organisms of higher tropic levels in the food chain that affects the non-target animals and mammals (Schauber et al. 1997; Dalkvist et al. 2009; Yadav 2010). The contamination of the water bodies such as ponds and the environment can indirectly affect human beings through its indirect source (George et al. 2004; Harris et al. 2010; Polson et al. 2011).

The biological control of mosquito larvae through entomopathogenic fungi is an alternative control strategy using Beauveria, Fusarium, Metarhizium and Aspergillus spp. of entomopathogenic fungi (Govindarajan et al. 2005).

Keeping in view the development of insecticidal resistance in mosquitoes, the current study has been designed to mycosynthesized AgNPs with locally isolated $F$. oxysporum and to investigate its anti-biological efficacy for the control of Aedes mosquito's larvae.

\section{Materials and Methods}

\section{Mosquito and fungal cultures}

The mosquito culture was maintained as described elsewhere (Vivekanandhan et al. 2018b). Aedes mosquitoes were briefly procured from the field and maintained in $1.5 \mathrm{~L}$ plastic containers containing tap water in the entomology laboratory, University of Veterinary and Animal Sciences, Lahore $\left(31.54972^{\circ} \mathrm{N}, 74.3436^{\circ} \mathrm{E}\right)$, Punjab, Pakistan. The larvae were fed with yeast powder as $3: 3: 1$ and kept in a range of temperatures from 25 to $35^{\circ} \mathrm{C}$ under a diurnal temperature regimen at relative humidity $(75-85 \%)$ under photoperiod 14:10 (Light and Dark) in a controlled chamber of Biological Oxygen Demand (BOD) incubator (Model ICO105 Memmert, Germany). The mosquito adults were identified by using the key described elsewhere (Rueda 2004). Adult Aedes mosquitoes were distinguished from other types of mosquitoes by their narrow and typically black body, unique patterns of light and dark scales on the abdomen and thorax, and alternating light and dark bands on the legs.

The soil samples were collected from the urbanized area of Lahore from 50 different locations. All soil samples were collected and processed as described elsewhere (Zhao et al. 2014). Fifty collected samples were initially subjected to air-drying. The soil samples were then passed through a fine sieve of $200 \mu \mathrm{m}$ capacity. Then, the aliquot of the sieved sample was spread over potato dextrose agar (PDA) and broth (PDB) at $27^{\circ} \mathrm{C}$ for seven days in a shaker incubator (Komada 1976). After the incubation, the fungal biomass was sieved through a sterilized cheese-cloth and washed twice using sterile distilled water to remove the excess medium components. $10 \mathrm{~g}$ of fungal biomass (wet weight) was mixed in $100 \mathrm{~mL}$ sterile double distilled water in an Erlenmeyer flask and incubated in a shaker incubator (RTSK-O300, Robus, U.K.) for $48 \mathrm{~h}$ at $120 \mathrm{rpm}$ and $28^{\circ} \mathrm{C}$. The aqueous solution components were again filtered with Whatman $^{\circledR}$ filter paper no.1 to get the mycelium-free 
filtrate. This mycelium-free filtrate was placed in a conical flask and mixed with $1 \mathrm{mM} \mathrm{AgNO} 3(0.017 \mathrm{~g} / 100 \mathrm{~mL})$ as the final concentration for reducing AgNPs. The mixer was again incubated in the shaker incubator under light at $28^{\circ} \mathrm{C}$ and $120 \mathrm{rpm}$. The mycelium-free filtrate without $\mathrm{AgNO}_{3}$ was considered to serve as a control and kept under the same conditions $\left(28^{\circ} \mathrm{C}\right.$ and $\left.120 \mathrm{rpm}\right)$. After $24 \mathrm{~h}$, the change in color of the reaction mixture treated with $\mathrm{AgNO}_{3}$ was observed, which was treated with $\mathrm{AgNO}_{3}$. After $120 \mathrm{~h}$ of incubation, the mycosynthesized AgNPs turned into a brownish yellow color solution. The AgNPs solution was stored in vials at $4^{\circ} \mathrm{C}$ until further characterizations. The positive control $F$. oxysporum (FCBP-PTF-0082) was acquired from the inventory of Fungal Culture Bank of Pakistan Institute of Agricultural Sciences, University of The Punjab, Lahore, Pakistan.

\section{Identification of fungus}

Based on the cultural characteristics and morphology, Fusarium colonies were identified (Leslie and Summerell 2008; Sever et al. 2012). The fungus was grown on PDA plates and identified under the microscope using the protocol described elsewhere (Nelson et al. 1983; Kostadinova et al. 2009). Briefly, adhesive tape was used to stick the fungus growth on a plate, and the tape was then attached to the glass slide to observe under the microscope (CX21FS1, Olympus, Japan) at 400X.

\section{DNA analysis}

A commercially available DNA extraction kit (GeneAll@, Exgene $\left.^{\mathrm{TM}}, 105-101\right)$ was used to acquire the purified DNA by following the manufacturer's instructions. The DNA was quantified using a Nano-drop spectrophotometer (Thermo Scientific, U.S.A.). Primers targeting the targeting Internal Transcribed Spacer (ITS) Sequence of F. oxysporum were adopted from a previous study FOF1: 5'ACATACCACTTGTTGCCTCG-3' and FOR1: 5'CGCCAATCAATTTGAGGAACG-3'(Mishra et al. 2003). PCR was performed according to the protocol described with little modifications (Mishra et al. 2003). Briefly, $20 \mu \mathrm{L}$ of PCR reaction was prepared for each sample, which included $10 \mu \mathrm{L}$ of $2 \mathrm{X}$ MasterMix (GeneAll $\left.{ }^{\circledR}\right), 1 \mu \mathrm{L}$ of each primer pair $(10 \mathrm{pmol}), 6 \mu \mathrm{L}$ of DEPC water (Invitrogen, U.S.A.) and $2 \mu \mathrm{L}$ of DNA. The annealing temperature was set as $60^{\circ} \mathrm{C}$ with 30 cycles each for the PCR reaction. The PCR was repeated 3 times for its optimization. The control positive and control negative were run for each reaction. Visualization of PCR product was done at GelDoc 100 imaging system after the electrophoresis $1.2 \%$ agarose gel stained with SYBR safe DNA gel stain (Invitrogen, USA). DNA molecular weight marker (Genedirex, Catalog \# DM001-R500) was used to compare the amplified product's size.

The PCR product was sequenced through Sanger Sequencing method (Men et al. 2008). The chromatograms were analyzed using the Chromas Pro software (version 1.7.4) and the sequences were compared with the GenBank database for its nucleotide sequence homology. The data search was done at the National Centre for Biotechnology Information (NCBI) network server with the BLAST algorithm. ITS Sequences of $F$. oxysporum from Genbank of NCBI were retrieved. They were aligned using CLUSTAL W (Thompson et al. 1994). Geneious R8.1.6 software (Kearse et al. 2012) was used for the alignment of sequence. A phylogenetic tree was constructed with a neighbor-joining method by using Geneious R8.1.6 software (Saitou and Nei 1987).

\section{Visual and ultra violet (UV)-visible (Vis) analysis}

$\mathrm{AgNO}_{3}$ was added to the fungal filtrate of $F$. oxysporum for the reduction of silver AgNPs, and the reaction mixture was kept in the light. The detection of AgNPs was primarily carried out by visualizing the color change of the fungal filtrate. The color of reaction mixture was changed gradually from pale yellow to brown, indicating the reduction of silver ions to AgNPs during $72 \mathrm{~h}$ of its incubation.

The formation of AgNPs was confirmed through UVVisible spectroscopic analysis. The AgNPs were characterized with the help of a UV-Vis spectrophotometer (Perkin-Elmer, Germany) by scanning the absorbance spectra in the wavelength range of $200-800 \mathrm{~nm}$ with water as a control.

\section{X-ray diffraction (XRD) analysis}

To confirm the crystalline structure of NPs, the mycelia-free fungal filtrate with AgNPs was lyophilized (FreeZone 2.5 Liter Benchtop Freeze Dryer, Catalogue No. 710202000, LABCON Co., Germany). This freeze-dried sample was analyzed by X'Pert PRO X-ray diffractometer using $\mathrm{Cu} \mathrm{K} \alpha$ radiation $(\lambda=1.54056 \AA)$ and operated at a voltage of $40 \mathrm{kV}$ for XRD analysis. The diffraction pattern was recorded in a scattering range $(2 \theta)$ of $10-80^{\circ}$.

\section{SEM, DLS and ZP analyses}

The biosynthesized AgNPs were investigated using SEM (Scanning electron microscopy). The morphology and nanostructure of AgNPs were analyzed with Nova NanoSEM 650 at $10 \mathrm{KV}$. The SEM micrographs were taken at 80,000x magnification. For SEM imaging, a thin film of AgNPs was prepared by drop coating of purified AgNPs from prepared solution onto carbon-coated copper SEM grid. The grid was allowed to evaporate for $5 \mathrm{~min}$. The surplus sample was removed using a blotting paper.

The size of AgNPs and ZP (Zeta Potential) was measured by laser diffractometer using a Nano-Size Particle Analyzer (ZEN 1600 MALVERN, USA) ranging from 0.1 $\mathrm{nm}$ to $10 \mu \mathrm{m}$ by following particle refractive index 1.33 , particle absorption coefficient 0.001 , water refractive index 
1.33 , viscosity $0.8872 \mathrm{cP}$, temperature $25^{\circ} \mathrm{C}$, count rate 259 Kcps and the calculation was done by using Malvern software (DTS, v. 7.10).

\section{Fourier-transform infrared spectroscopy (FTIR) analysis}

After the complete reduction of silver ions into AgNPs, the characterization of functional groups of AgNPs was done by FTIR using (Perkin-Elmer, Germany). The spectra were scanned in the $400-4,000 \mathrm{~cm}^{-1}$ range with a resolution of 4 $\mathrm{cm}^{-1}$. FTIR measurements of the samples identified the probable interactions between silver and the bio-active molecules, which may be essential for the reduction of $\mathrm{Ag}^{+}$ to $\mathrm{Ag}^{0}$ and stabilization (capping material) of AgNPs. A small sample was mixed with $100 \mathrm{mg} \mathrm{KBr}$ and a pellet was made by pressing with a pellet making machine. The background calibrations were carried out using a pure $\mathrm{KBr}$ pellet. The observed peaks were plotted as percentages taking the transmittance along the $\mathrm{x}$-axis and wave number along the y-axis.

\section{Larvicidal assay}

The larvicidal activities of $F$. oxysporum with or without AgNPs on Aedes larvae were assessed as per the method mentioned elsewhere (WHO 2018). Cypermethrin was used as control positive and sterilized distilled water was used as control negative. Concentration $(1.0 \mathrm{ppm})$ was placed on the fourth instars of Aedes mosquitoes (Banu and Balasubramanian 2014a). 15 larvae of Aedes mosquitoes were taken in $250 \mathrm{~mL}$ plastic containers having $150 \mathrm{~mL}$ distilled water. The mortality of the larvae was noted after every hour during the experimental period ( $24 \mathrm{~h})$. Larval mortalities (WHO 1981) were recorded after every hour (Banu and Balasubramanian 2014b; Banu et al. 2014) and data was corrected by using Abbott's formula (Abbott 1925). The mean larval mortality was calculated at the regular interval from the three replicates.

\section{Statistical analysis}

The mortality rate was calculated in the larvicidal assay (Mahnaz et al. 2012). A curve for larval mortality was prepared and statistical analysis (Log rank test for trend) was done using Graph Pad Prism Version 7 (Magro et al. 2019).

\section{Results}

\section{Identification and phylogenetic analysis of $F$. oxysporum}

The morphology of the fungus was observed through a microscope and its genetic structure was confirmed through PCR. Product size; 339 bp was obtained and control positive DNA (339 bp) for $F$. oxysporum, as

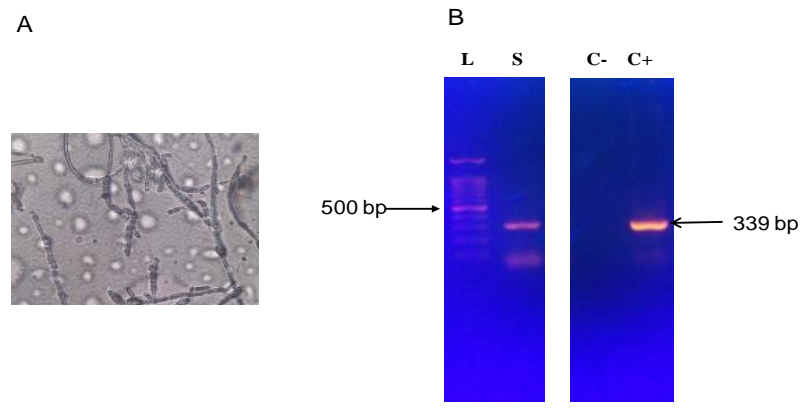

Fig. 1: Identification of $F$. oxysporum. (A) Morphology, (B) PCR; $\mathrm{L}$ is DNA ladder, $\mathrm{S}$ is a sample, $\mathrm{C}+$ is control positive DNA and $\mathrm{C}$ - is control negative DNA

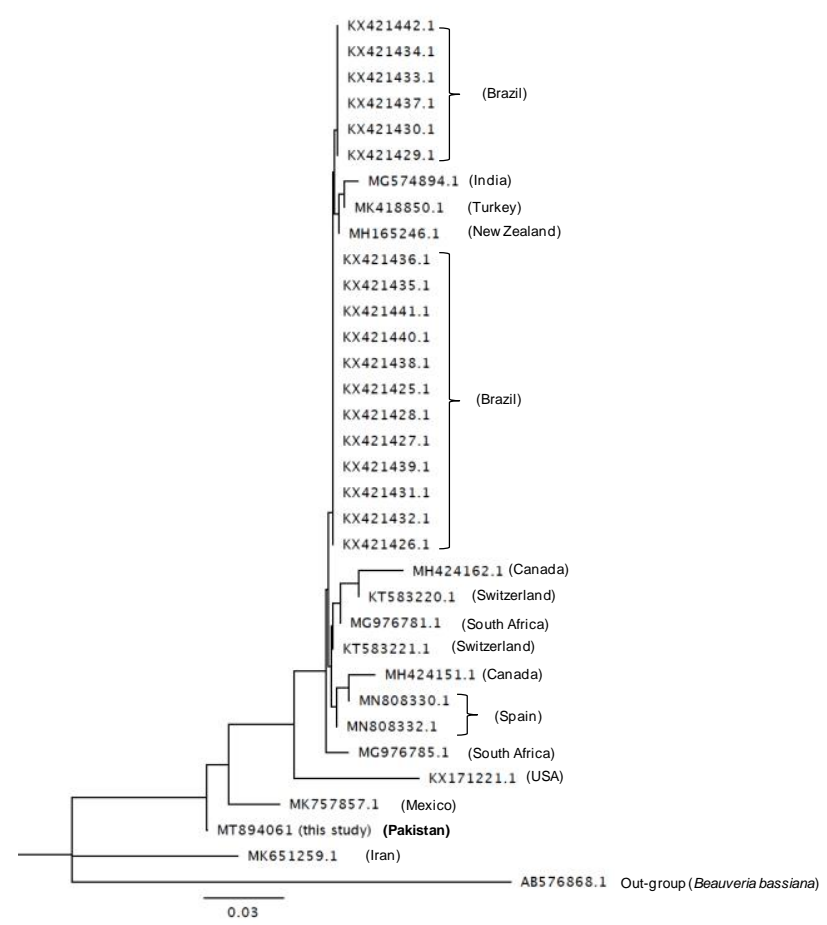

Fig. 2: Phylogenetic analysis of $F$. oxysporum on the basis of Internal Transcribed Spacer sequence. Our sequence (MT894061) was closely related to the sequence from Mexico (MK757857). AB576868 was taken as an out-group from Beauveria bassiana. Pakistan is highlighted bold before our sequence. The sequence alignment and phylogenetic sequence construction was done with Geneious R8.1.6 software and the other sequences were retrieved from Genbank database of National Center for Biotechnology Information (NCBI)

shown in Fig. 1. The PCR products were also confirmed through sequencing. In the BLAST analysis, 99\% homology was observed with ITS DNA of $F$. oxysporum from other parts of the world. The phylogenetic analysis of our sequence (MT894061) was done as shown in Fig. 2. Our sequence (MT894061) was placed in the clade with MK651259.1. 

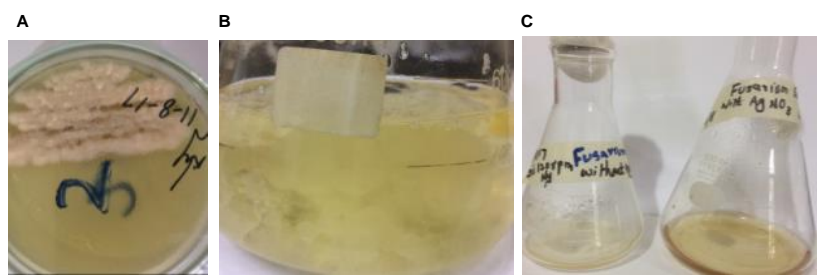

Fig. 3: F. oxysporum culture on agar plate (A), in broth (B) and its mycosynthesis in nanoparticles $(\mathbf{C})$

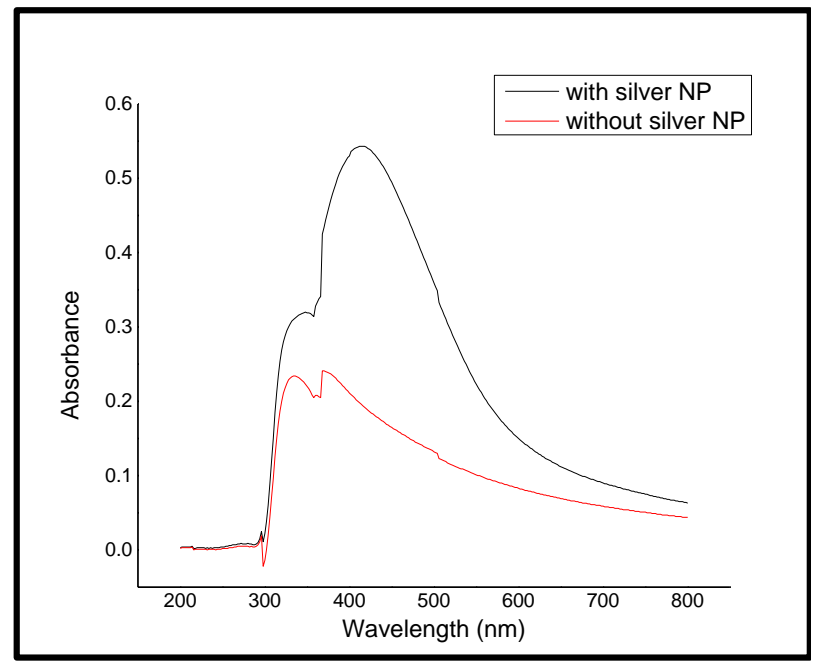

Fig. 4: Ultraviolet-visible absorption spectrum AgNPs synthesized by Entomopathogenic fungus; $F$. oxysporum.

\section{XRD analysis of Nanoparticles}

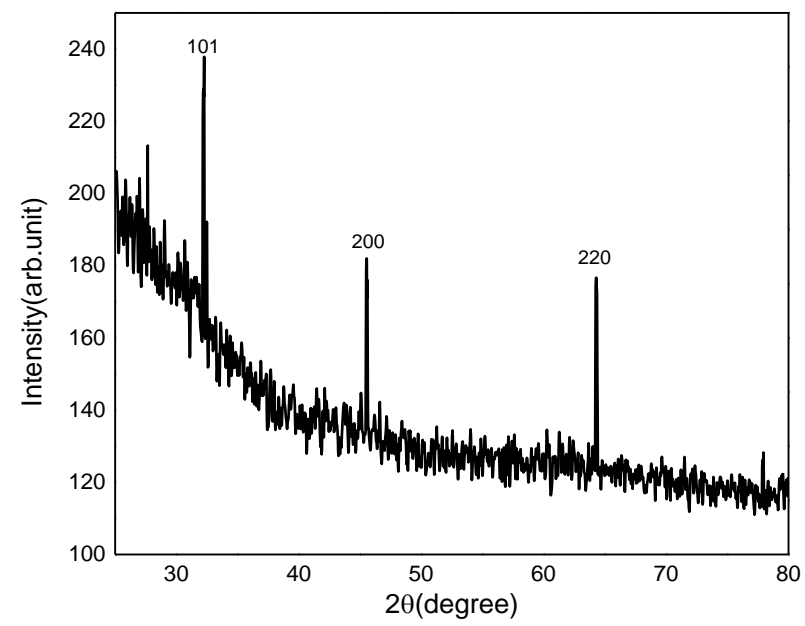

Fig. 5: X-ray diffraction pattern of $1 \mathrm{~m} M$ AgNPs synthesized by F. oxysporum. The peaks $(101,200,220)$ showed the crystalline phases of silver (Ag)

\section{Culture and myco-synthesis of Fusarium NPs}

Crystal white growth of $F$. oxysporum was observed on the agar and broth of the medium as shown in Fig. 3A and 3B.
The filtered fungal residues were apparent (without NPs and dark brown (with NPs) after incubation at $28^{\circ} \mathrm{C}$ for $48 \mathrm{~h}$, as shown in Fig. 3C.

\section{UV-Vis analysis}

The UV-Vis spectra of fungal filtrate with AgNPs or without AgNPs is shown in Fig. 4, a broad surface Plasmon peak was observed at $420 \mathrm{~nm}$ wavelength while a narrow peak occurred at $310 \mathrm{~nm}$ after $72 \mathrm{~h}$ of incubation.

\section{XRD}

Inspection of the X-Ray diffraction patterns of NPs revealed the existence of sharp diffraction peaks at $2 \theta$ angles of $32.19^{\circ}, 45.55^{\circ}$ and $64.27^{\circ}$ that can be indexed to the (101), (200) and (220) facets of Ag which were in line with the values reported for Face-centered cubic (fcc) lattice of AgNPs in Joint Committee on Powder Diffraction Standard card as shown in Fig. 5.

\section{SEM, DLS and ZP analyses}

The Scanning Electron Microscopy (SEM) micrograph showed well-defined spherical NPs which were smooth, isotropic (i.e., with low aspect ratio) and poly-dispersed. The observed size of AgNPs ranged from 10-200 nm. The micrograph showed the agglomeration of NPs. The NPs were not in direct contact with each other even in the aggregate form, thus; indicating the stabilization of AgNPs by a capping agent present in the fungal filtrate as shown in Fig. 6A.

The size distribution by intensity profile determined by Dynamic Light Scattering (DLS) showed unimodal distribution. The average diameter of AgNPs determined by DLS is $167 \mathrm{~nm}$ with the poly-disparity index of 0.16 . The AgNPs showed a Zeta Potential (ZP) of $-4.78 \mathrm{mV}$. These ZP measurements and poly-disparity index of 0.16 by DLS revealed reduced size variability and a sound physio-chemical stability of biosynthesized AgNPs as shown in Fig. 6B, C.

\section{FTIR analysis}

The FTIR spectrum of biosynthesized AgNPs showed strong bands at 3280 and $1635 \mathrm{~cm}^{-1}$ as shown in Fig. 7A. Some weak bands were also recorded in the wave number range of 1900 to $2200 \mathrm{~cm}^{-1}$ as shown in Fig. 7B.

\section{Larvicidal Assay of F. oxysporum AgNPs}

Mosquito larvae were observed for $24 \mathrm{~h}$ after exposure, as shown in Fig. 8. The rate of mortality of mosquitoe's larvae was recorded, as shown in Fig. 9. The mosquitoes' mortality was found highly significant $(P<0.001)$ in the group treated with Fusarium NPs compared to the negative control group. 


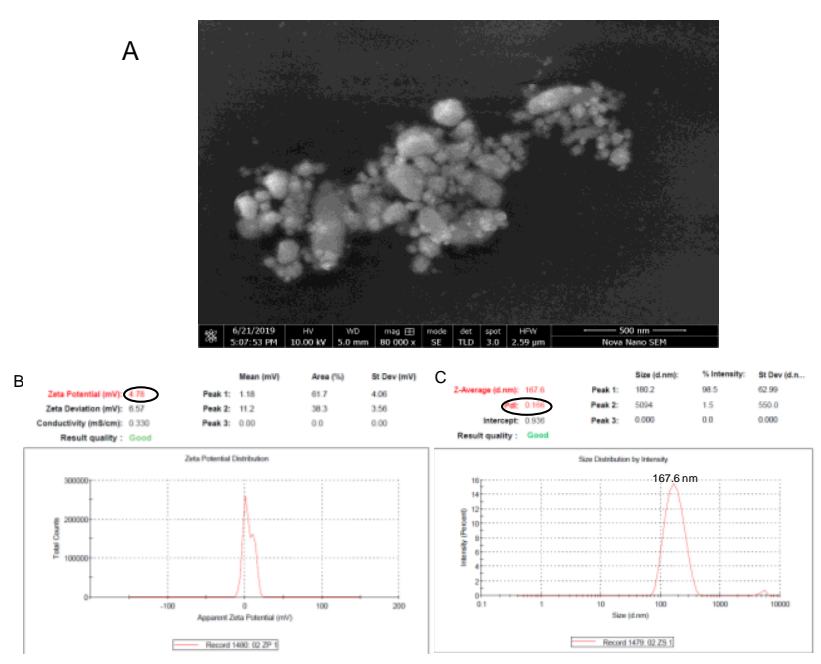

Fig. 6: SEM micrographs of AgNPs showing spherical shapes of AgNPs (A), and Dynamic Light Scattering (DLS) measurements for Zeta potential analysis showing the stability of AgNPs $(\mathbf{B})$ and DLS measurements for particle size distribution showing 167.6 $\mathrm{nm}$ average particle size $(\mathbf{C})$

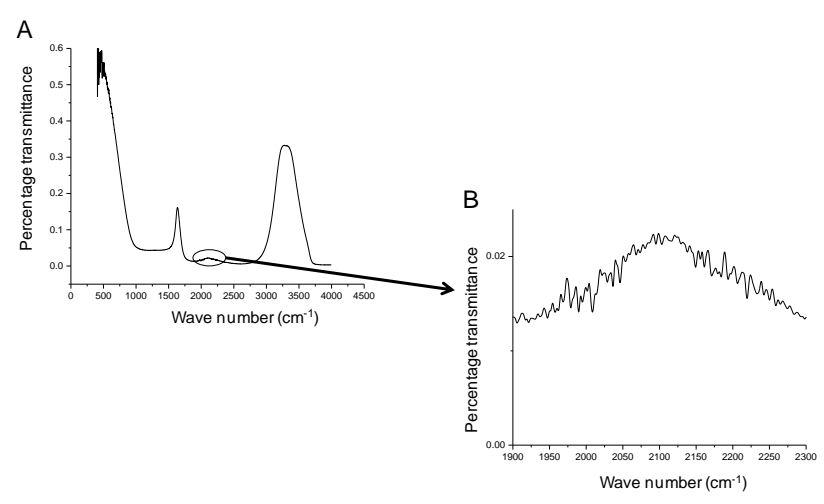

Fig. 7: Fourier-transform infrared spectroscopy. Wave number ranges from 0 to $4500 \mathrm{~cm}^{-1}$ on the $\mathrm{x}$-axis and \%age transmittance from 0 to 0.6 on the $y$-axis (A). Magnified image of A, Wave number ranges from 1900 to $2300 \mathrm{~cm}^{-1}$ on $\mathrm{x}$-axis and \% transmittance from 0 to 0.25 on the y-axis $(\mathbf{B})$

The larvae exposed to Fusarium without NPs survived $7 \mathrm{~h}$ longer than the larvae of mosquitoes exposed to Fusarium with NPs.

\section{Discussion}

Previously, F. oxysporum derived AgNPs have enhanced entomopathogenic activity against Culex mosquito larvae (Vivekanandhan et al. 2018b). We have isolated a fungus from the soil in Lahore, Pakistan through PCR, which has phylogenetic similarity with the strain of $F$. oxysporum isolated from the body of Aedes aegypti (Aguirre-Joya et al. 2018). Moreover, we have characterized our NPs through UV-Vis, SEM, DLS, ZP and FTIR. We have observed $100 \%$ mortality of Aedes mosquitoes larvae at $17 \mathrm{~h}$ and $24 \mathrm{~h}$
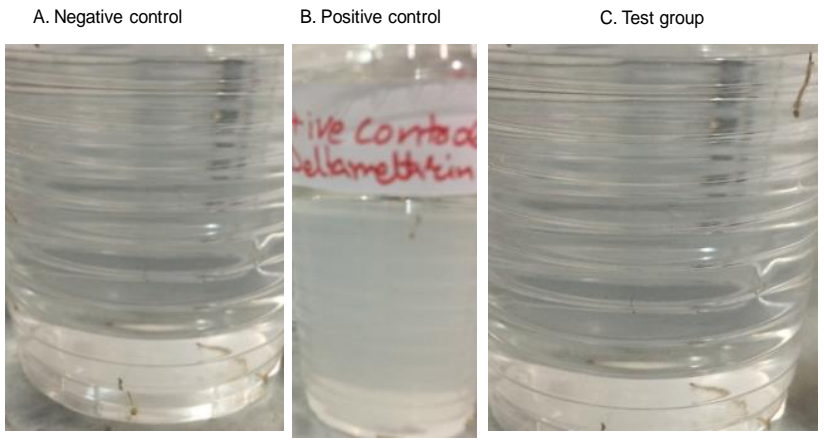

Fig. 8: Anti-biological activity of AgNPs of $F$. oxysporum. Control negative $(\mathbf{A})$ which was without any treatment. Control positive (B), which was treated with Cypermethrin. Test group (C) which was treated with fungal AgNPs

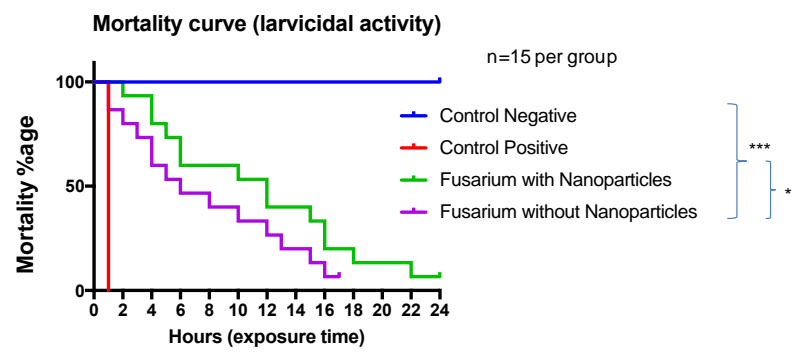

Fig. 9: Mortality curve showing the mortality rate of the larvae of Aedes mosquitoes. Control negative was distilled water and control positive was cypermethrin

with AgNPs and without AgNPs by using $1 \mathrm{ppm}$ concentration, respectively.

UV-Vis spectroscopy could be availed to examine the size and shape-controlled NPs in the aqueous suspensions (Fayaz et al. 2009). Biosynthesized AgNPs exhibit absorption peaks at a wavelength range of $410-445 \mathrm{~nm}$ (Durán et al. 2005; Ashrafi et al. 2013). The yellowishbrown color appearance was an indication of the formation of AgNPs in the medium. The brown color could mainly be due to Surface Plasmon Vibrations' excitation, typical of the AgNPs correlated with the results obtained by (Husseiny et al. 2015). They reported the extracellular biosynthesis of AgNPs using $F$. oxysporum with UV-Vis absorption peak at $420 \mathrm{~nm}$. There was no absorption band at $420 \mathrm{~nm}$ in the control sample's UV-Vis spectra after 72 incubation hours. An absorption band at $310 \mathrm{~nm}$ wavelength was visible, and it is attributed to aromatic amino acids of proteins present in the fungal filtrate (Durán et al. 2005; Jain et al. 2011). This absorption band indicated the release of protein into the fungal filtrate, suggesting the possible mechanism for reducing silver ions present in the solution.

The resultant XRD spectrum analysis suggested that biosynthesized AgNPs from the entomopathogenic fungus. $F$. oxysporum were crystalline and the same was evaluated through SEM. The diffraction peaks corresponding to (101), (200) and (220) due to broadening of Bragg reflections at 
$32.19^{\circ}, 45.55^{\circ}$ and $64.27^{\circ}$, respectively showed the FCC cubic structure of AgNPs and are in agreement with similarly reported peaks from the literature (Musarrat et al. 2010). A similar XRD diffraction pattern was reported by Ashrafi et al. (2013) in size and shape controllable biofabrication of silver nano-crystallites using the growth extract of the fungus Rhizoctonia solani. They observed Bragg reflections at 38.1, 41.3 and 58.6, respectively based on the face-centered cubic structure (Ashrafi et al. 2013).

In the present study, no peak of the XRD pattern of silver with other substances was observed, indicating that NPs contain silver in high purity. The observed noise in the XRD pattern was probably due to the effect of nano-sized particles and various crystalline biological molecules in the fungal filtrate. The observed results showed that silver was reduced by fungal extract and was present in high purity with face-centered cubic structure in NPs.

FTIR spectroscopy is a powerful tool for quantifying secondary structure in metal NPs and protein interaction. In the literature, all the FTIR spectra of biosynthesized AgNPs confirmed the protein capping on AgNPs. The presence of all bands between 3300 and $3500 \mathrm{~cm}^{-1}$ in all FTIR spectra showed the N-H stretching of amide A band and $\mathrm{O}-\mathrm{H}$ stretching of aromatic amines (Hamedi et al. 2014; Gurunathan 2019). These bands showed strong hydrogen bonding on the boundary of NPs, while all peaks between 1630 and $1680 \mathrm{~cm}^{-1}$ in all FTIR spectra arise from $\mathrm{C}=\mathrm{O}$ (carbonyl) stretch vibrations in the amide I and amide II bonds of proteins (Birla et al. 2013; Joshi et al. 2013). In the present study, the FTIR spectra of AgNPs synthesized by entomopathogenic $F$. oxysporum, showed a sharp absorption peak at $3280 \mathrm{~cm}^{-1}$ associated with $\mathrm{O}-\mathrm{H}$ stretch hydroxyl group of protein. Another band at $1635 \mathrm{~cm}^{-1}$ has been identified as an amide band that arose due to $\mathrm{C}=\mathrm{O}$ (carbonyl) stretch vibrations in the amide linkage of protein. It is a well-known mycoprotein property secreted out to bind through free amine groups or cysteine residues. It can also bind through the electrostatic attraction of negatively charged carboxylate groups in enzymes present in mycelia's cell wall and therefore, stabilizes the AgNPs in the solution (Gajbhiye et al. 2009). Our observations support the findings of previous literature. These vibrational bands' positions are close to the results of Vahabi et al. (2011), they reported the strong absorption bands at 3450 and $1642 \mathrm{~cm}^{-1}$ while weak bands were obtained at 1516, 1455 and 1074 $\mathrm{cm}^{-1}$. It was concluded that the secondary structure of proteins was not affected because of its interaction with $\mathrm{Ag}^{+}$ ions or NPs (Vahabi et al. 2011). Similar results were obtained in extracellular biosynthesis of AgNPs by using $F$. oxysporum (Ahmad et al. 2003). They reported the presence of three FTIR bands at 1650, 1540 and $1450 \mathrm{~cm}^{-1}$. The exact mechanism behind reducing silver ions to AgNPs is yet to be elucidated for $F$. oxysporum. From FTIR analysis, we can infer that the carbonyl group from amino acid residues binds the NPs and plays a pivotal role in reducing silver ions to AgNPs and stabilizing in aqueous solution.
The present observations in SEM micrographs corroborate with Banu et al. (2014). They synthesized the AgNPs with the help of entomopathogenic Isaria fumosorosea and reported well defined and spherical shaped NPs ranging from $51.31 \mathrm{~nm}$ to $111.02 \mathrm{~nm}$ (Banu and Balasubramanian 2014b; Banu et al. 2014). F. oxysporum derived AgNPs were in size range from $20 \mathrm{~nm}$ to $50 \mathrm{~nm}$ which were also reported by Durán et al. (2005). They observed AgNPs aggregates in SEM micrographs and confirmed the presence of protein capping on NPs (Durán et al. 2005). Similar observations were reported by other researchers in their studies on AgNPs synthesized from Aspergillus niger cell-free filtrate. They reported the agglomerated AgNPs having a size ranging from $70 \mathrm{~nm}$ to 200 nm (Nithya and Ragunathan 2014).

The present study results are in agreement with the findings of (Guilger et al. 2017). They reported the biogenic AgNPs based on Trichoderma harzianum which showed a $\mathrm{ZP}$ of $-6.85 \pm 1.45 \mathrm{mV}$ by micro electrophoresis technique and a polydispersity index of $0.27 \pm 0.03$ by DLS. The application of the biological stabilizers found in the fungal filtrate has been reported earlier in the literature to possess the ability to stabilize and improve the dispersion of the NPs(Gade et al. 2008).

Previously, entomopathogenic fungi such as Beauveria bassiana (Banu and Balasubramanian 2014a; Tyagi et al. 2019) Isaria fumosorosea (Banu and Balasubramanian 2014b), Chrysosporium tropicum (Soni and Prakash 2012a), Trichoderma harzianum (Sundaravadivelan and Padmanabhan 2014) Aspergillus niger (Soni and Prakash 2012b) and F. oxysporum (Vivekanandhan et al. 2018a) had been used against Aedes mosquitoes for their larvicidal activities. These entomopathogenic fungi has toxic metabolites cause damage to the cuticle of mosquito larvae (Mannino et al. 2019). Banu and Balasubramanian (2014) attained 52.4, $60.0,68.5,76.0$ and $83.3 \%$ mortality at $24 \mathrm{~h}$ of exposure in 0.06 and $1.00 \mathrm{ppm}$ of Beauveria bassiana NPs against Ae. Aegypti. Some researchers observed larvicidal Activity of AgNPs synthesized using extracts of Ambrosia arborescens to control Ae. aegypti (Morejón et al. 2018). They observed $96.4 \pm 1.8$ mortality in third instar larvae by using $0.5 \mathrm{ppm}$. The difference of mortality in our study with other studies might be due to the use of different entomopathogenic plants. Our study showed highest mortality (100\%) of Aedes larvae at $17 \mathrm{~h}$ and $24 \mathrm{~h}$ after exposure of $1 \mathrm{ppm}$ concentration of NPs with AgNPs and without AgNPs, respectively. Vivekanandhan et al. (2018a) achieved strong mortality against Ae. aegypti larvae by using $F$. oxysporum AgNPs. The nanoparticles of different fungi have different effects on mosquito larvae. Fusarium AgNPs prepared through different methods have different results as shown by Vivekanandhan et al. (2018b) who used dark condition and Sabourand's Dextrose broth for the synthesis of Fusarium AgNPs. 


\section{Conclusion}

F. oxysporum NPs enhanced the anti-biological activity by causing mortality in Aedes larvae $7 \mathrm{~h}$ earlier than $F$. oxysporum without NPs. Therefore, Fusarium AgNPs could be an alternative to chemicals against mosquito larvae.

\section{Acknowledgements}

The authors are thankful to Dr. Muhammad Rashid, Lanzhou Veterinary Research Institute, Chinese Academy of Agricultural Sciences, China, for the analyses of samples.

\section{Author Contributions}

NSS, ZA and STK collected the samples, carried out the experiment, performed the analysis and drafted the manuscript. WS and SSI designed the study, performed analysis and reviewed the article.

\section{Conflicts of Interest}

All authors declare that there is no conflict of interest

\section{Data Availability}

Data presented in this study will be available on a fair request to the corresponding author

\section{Ethics Approval}

Not applicable in this paper

\section{Refernces}

Abbott WS (1925). A Method of computing the effectiveness Of an insecticide. J Econ Entomol 18:265-267

Abdel-Aziz MM, M Yosri, BH Amin (2017). Control of imipenem resistant-Klebsiella pneumoniae pulmonary infection by oral treatment using a combination of mycosynthesized Ag-nanoparticles and imipenem. J Radiat Res Appl Sci 10:353-360

Aguirre-Joya JA, L Pastrana-Castro, D Nieto-Oropeza, J VenturaSobrevilla, R Rojas-Molina, CN Aguilar (2018). The physicochemical, antifungal and antioxidant properties of a mixed polyphenol based bioactive film. Heliyon 4; Article e00942

Ahmad A, P Mukherjee, S Senapati, D Mandal, MI Khan, R Kumar, M Sastry (2003). Extracellular biosynthesis of silver nanoparticles using the fungus Fusarium oxysporum. Colloids Surf B Biointerf 28:313-318

Ashrafi SJ, MF Rastegar, M Ashrafi, F Yazdian, R Pourrahim, AK Suresh (2013). Influence of external factors on the production and morphology of biogenic silver nanocrystallites. $J$ Nanosci Nanotechnol 13:2295-2301

Banu AN, C Balasubramanian (2014a). Myco-synthesis of silver nanoparticles using Beauveria bassiana against dengue vector, Aedes aegypti (Diptera: Culicidae). Parasitol Res 113:2869-2877

Banu AN, C Balasubramanian (2014b). Optimization and synthesis of silver nanoparticles using Isaria fumosorosea against human vector mosquitoes. Parasitol Res 113:3843-3851

Banu AN, C Balasubramanian, PV Moorthi (2014). Biosynthesis of silver nanoparticles using Bacillus Thuringiensis against dengue vector, Aedes aegypti (Diptera: Culicidae). Parasitol Res 113:311-316
Bar H, DK Bhui, GP Sahoo, P Sarkar, SP De, A Misra (2009). Green synthesis of silver nanoparticles using latex of Jatropha curcas. Colloids Surf A Physicochem Eng Asp 339:134-139

Becker RO (1999). Silver ions in the treatment of local infections. MetalBased Drugs 6:311-314

Birla SS, SC Gaikwad, AK Gade, MK Rai (2013). Rapid synthesis of silver nanoparticles from Fusarium oxysporum by optimizing physicocultural conditions. Sci World J 2013; Article 796018

Chakraborty I, T Pradeep (2017). Atomically precise clusters of noble metals: Emerging link between atoms and nanoparticles. Chem Rev 117:8208-8271

Colvin V, M Schlamp, AP Alivisatos (1994). Light-emitting-diodes made from cadmium selenide nanocrystals and a semiconducting polymer. Nature 370:354-357

Crabtree JH, RJ Burchette, RA Siddiqi, IT Huen, LL Hadnott, A Fishman (2003). The efficacy of silver-ion implanted catheters in reducing peritoneal dialysis-related infections. Perit Dial Intl 23:368-374

Dalkvist T, CJ Topping, VE Forbes (2009). Population-level impacts of pesticide-induced chronic effects on individuals depend more on ecology than toxicology. Ecotoxicol Environ Saf 72:1663-1672

Devi LS, S Joshi (2015). Ultrastructures of silver nanoparticles biosynthesized using endophytic fungi. $J$ Microsc Ultrastruct 3:29-37

Dolgaev S, A Simakin, V Voronov, GA Shafeev, F Bozon-Verduraz (2002). Nanoparticles produced by laser ablation of solids in liquid environment. Appl Surf Sci 186:546-551

Durán N, PD Marcato, GID Souza, OL Alves, E Esposito (2007). Antibacterial effect of silver nanoparticles produced by fungal process on textile fabrics and their effluent treatment. J Biomed Nanotechnol 3:203-208

Durán N, PD Marcato, OL Alves, GID Souza, E Esposito (2005). Mechanistic aspects of biosynthesis of silver nanoparticles by several Fusarium oxysporum strains. J Nanobiotechnol 3:1-7

Dyal C, N Nguyen, J Hadden, L Gou, L Tan, CJ Murphy, W Lynch, D Nivens (2006). Green synthesis of gold and silver nanoparticles from plant extracts. Chem Mater 26:301-315

Evanoff DD, G Chumanov (2004). Size-controlled synthesis of nanoparticles. 2. measurement of extinction, scattering, and absorption cross sections. J Phys Chem B 108:13957-13962

Fayaz AM, K Balaji, P Kalaichelvan, R Venkatesan (2009). Fungal based synthesis of silver nanoparticles - an effect of temperature on the size of particles. Colloids Surf B Biointerf 74:123-126

Fradin MS, JF Day (2002). Comparative efficacy of insect repellents against mosquito bites. Eng J Med 347:13-18

Gade A, P Bonde, A Ingle, P Marcato, N Duran, M Rai (2008). Exploitation of Aspergillus niger for synthesis of silver nanoparticles. J Biobased Mater Bioener 2:243-247

Gajbhiye M, J Kesharwani, A Ingle, A Gade, M Rai (2009). Fungusmediated synthesis of silver nanoparticles and their activity against pathogenic fungi in combination with fluconazole. Nanomed Nanotechnol Biol Med 5:382-386

George J, J Pound, R Davey (2004). Chemical control of ticks on cattle and the resistance of these parasites to acaricides. Parasitology 129:353-366

Giner-Casares JJ, M Henriksen-Lacey, M Coronado-Puchau, LM LizMarzán (2016). Inorganic nanoparticles for biomedicine: Where materials scientists meet medical research. Mater Today 19:19-28

Govindarajan M, A Jebanesan, D Reetha (2005). Larvicidal effect of extracellular secondary metabolites of different fungi against the mosquito, Culex quinquefasciatus say. Trop Biomed 22:1-3

Guilger M, T Pasquoto-Stigliani, N Bilesky-Jose, R Grillo, P Abhilash, LF Fraceto, R De Lima (2017). Biogenic silver nanoparticles based on Trichoderma harzianum: Synthesis, characterization, toxicity evaluation and biological activity. Sci Rep 7; Article 44421

Gurunathan S (2019). Rapid biological synthesis of silver nanoparticles and their enhanced antibacterial effects against Escherichia fergusonii and Streptococcus mutans. Arab J Chem 12:168-180 
Hamedi S, SA Shojaosadati, S Shokrollahzadeh, S Hashemi-Najafabadi (2014). Extracellular biosynthesis of silver nanoparticles using a novel and non-pathogenic fungus, Neurospora intermedia: Controlled synthesis and antibacterial activity. World J Microbiol Biotechnol 30:693-704

Harris AF, S Rajatileka, H Ranson (2010). Pyrethroid resistance in Aedes aegypti from grand cayman. Amer J Trop Med Hyg 83:277-284

Husseiny SM, TA Salah, HA Anter (2015). Biosynthesis of size controlled silver nanoparticles by Fusarium oxysporum, their antibacterial and antitumor activities. Beni-Suef J Basic Appl Sci 4:225-231

Jain N, A Bhargava, S Majumdar, J Tarafdar, J Panwar (2011). Extracellular biosynthesis and characterization of silver nanoparticles using Aspergillus flavus NJP08: A mechanism perspective. Nanoscale 3:635-641

Jiang H, S Manolache, ACL Wong, FS Denes (2004). Plasma-enhanced deposition of silver nanoparticles onto polymer and metal surfaces for the generation of antimicrobial characteristics. J Appl Polym 93:1411-1422

Joshi P, S Bonde, S Gaikwad, A Gade, K Abd-Elsalam, M Rai (2013). Comparative studies on synthesis of silver nanoparticles by Fusarium oxysporum and Macrophomina phaseolina and it's efficacy against bacteria and malassezia furfur. $J$ Bionanosci 7:378-385

Kabashin AV, M Meunier (2003). Synthesis of colloidal nanoparticles during femtosecond laser ablation of gold in water. $J$ Appl Phys 94:7941-7943

Kearse M, R Moir, A Wilson, S Stones-Havas, M Cheung, S Sturrock, S Buxton, A Cooper, S Markowitz, C Duran, T Thierer, B Ashton, P Meintjes, A Drummond (2012). Geneious basic: An integrated and extendable desktop software platform for the organization and analysis of sequence data. Bioinformatics 28:1647-1649

Khan I, K Saeed, I Khan (2019). Nanoparticles: Properties, applications and toxicities. Arab J Chem 12:908-931

Komada H (1976). A new selective medium for isolating Fusarium from natural soil. In: Proceedings of American Phytopathological Society, Vol. 3, p:221. St. Paul, Minnesota, USA

Kostadinova N, E Krumova, S Tosi, Pashova, M Angelova (2009). Isolation and identification of filamentous fungi from island livingston, antarctica. Biotechnol Biotechnol Equipm 23:267-270

Leslie JF, BA Summerell (2008). The Fusarium laboratory manual. John Wiley \& Sons, New York, USA

Liu N (2015). Insecticide resistance in mosquitoes: Impact, mechanisms, and research directions. Annu Rev Entomol 60:537-559

Magro M, S Bramuzzo, D Baratella, J Ugolotti, G Zoppellaro, G Chemello, I Olivotto, C Ballarin, G Radaelli, B Arcaro (2019). Selfassembly of chlorin-e6 on $\gamma-\mathrm{Fe}_{2} \mathrm{O}_{3}$ nanoparticles: Application for larvicidal activity against Aedes aegypti. J Photochem Photobiol 194:21-31

Mahnaz K, F Alireza, V Hassan, S Mahdi, AM Reza, H Abbas (2012). Larvicidal activity of essential oil and methanol extract of Nepeta menthoides against malaria vector Anopheles stephensi. Asian Pac J Trop Med 5:962-965

Mannino MC, C Huarte-Bonnet, B Davyt-Colo, N Pedrini (2019). Is the insect cuticle the only entry gate for fungal infection? insights into alternative modes of action of entomopathogenic fungi. J Fung 5; Article 33

Mbanzulu KM, LE Mboera, FK Luzolo, R Wumba, G Misinzo, SI Kimera (2020). Mosquito-borne viral diseases in the democratic republic of the Congo: A review. Parasit Vect 13; Article 103

Mcnair CM (2015). Ectoparasites of medical and veterinary importance: Drug resistance and the need for alternative control methods. $J$ Pharm Pharmacol 67:351-363

Men AE, P Wilson, K Siemering, S Forrest (2008). Sanger DNA sequencing. In: Next Generation Genome Sequencing: Toward Personalized Medicine, pp:1-11. Wiley-VCH Verlag GmbH \& Co. $\mathrm{KGaA}$, Weinheim, Germany

Mishra PK, RT Fox, A Culham (2003). Development of a PCR-based assay for rapid and reliable identification of pathogenic Fusaria. FEMS Microbiol Lett 218:329-332
Morejón B, F Pilaquinga, F Domenech, D Ganchala, A Debut, M Neira (2018). Larvicidal activity of silver nanoparticles synthesized using extracts of Ambrosia arborescens (Asteraceae) to control Aedes aegypti 1.(Diptera: Culicidae). J Nanotechnol 2018; Article 6917938

Mubbashir H, S Munir, R Kashif, HB Nawaz, B Abdul, K Baharullah (2018). Characterization of dengue virus in Aedes aegypti and Aedes albopictus spp. of mosquitoes: A study in Khyber Pakhtunkhwa, Pakistan. Mol Biol Res Commun 7:77-82

Musarrat J, S Dwivedi, BR Singh, AA Al-Khedhairy, A Azam, A Naqvi (2010). Production of antimicrobial silver nanoparticles in water extracts of the fungus Amylomyces rouxii strain KSU-09. Bioresour Technol 101:8772-8776

Nagajyothi P, SP Vattikuti, K Devarayapalli, K Yoo, J Shim, T Sreekanth (2020). Green synthesis: Photocatalytic degradation of textile dyes using metal and metal oxide nanoparticles-latest trends and advancements. Crit Rev Environ Sci Technol 50:2617-2723

Nauen R (2007). Insecticide resistance in disease vectors of public health importance. Pest Manage Sci Form Pest Sci 63:628-633

Nelson PE, TA Toussoun, W Marasas (1983). Fusarium Species: An Illustrated Manual For Identification. Pennsylvania State University Press, Pennsylvania, USA

Nithya R, R Ragunathan (2014). In vitro synthesis, characterization and medical application of silver nanoparticle by using a lower fungi. Middle-East J Sci Res 21:922-928

Polson KA, WG Brogdon, SC Rawlins, DD Chadee (2011). Characterization of insecticide resistance in trinidadian strains of Aedes aegypti mosquitoes. Acta Trop 117:31-38

Qureshi EMA, AB Tabinda, S Vehra (2017). Seasonal and spatial quantitative changes in Aedes aegypti under distinctly different ecological areas of Lahore, Pakistan. J Pak Med Assoc 67:1797-1802

Rai M, A Yadav, A Gade (2009). Silver nanoparticles as a new generation of antimicrobials. Biotechnol Adv 27:76-83

Rana SM, EA Khan, A Yaqoob, AA Latif, MM Abbasi (2014). Susceptibility and irritability of adult forms of main malaria vectors against insecticides used in the indoor residual sprays in Muzaffargarh district, Pakistan: A field survey. J Med Entomol $51: 387-391$

Ranson H, J Burhani, N Lumjuan, WC Black (2010). Insecticide resistance in dengue vectors. Trop Net 1:1-7

Ray PC (2010). Size and shape dependent second order nonlinear optical properties of nanomaterials and their application in biological and chemical sensing. Chem Rev 110:5332-5365

Roco MC (2003). Nanotechnology: Convergence with modern biology and medicine. Curr Opin Biotechnol 14:337-346

Rueda LM (2004). Pictorial keys for the identification of mosquitoes (Diptera: Culicidae) associated with dengue virus transmission. In Zootaxa, pp:1-61. Magnolia Press, Auckland, New Zealand

Saitou N, M Nei (1987). The neighbor-joining method: A new method for reconstructing phylogenetic trees. Mol Biol Evol 4:406-425

Samish M, G Gindin, E Alekseev, I Glazer (2001). Pathogenicity of entomopathogenic fungi to different developmental stages of Rhipicephalus sanguineus (Acari: Ixodidae). $J$ Parasitol 87:1355-1359

Schauber EM, WD Edge, JO Wolff (1997). Insecticide effects on small mammals: Influence of vegetation structure and diet. Ecol Appl 7:143-157

Sever Z, D Ivić, T Kos, T Miličević (2012). Identification of Fusarium species isolated from stored apple fruit in croatia. Arh Higij Rada Toksikol 63:463-469

Simonsen PE, ME Mwakitalu (2013). Urban lymphatic filariasis. Parasitol Res 112:35-44

Soni N, S Prakash (2012a). Efficacy of fungus mediated silver and gold nanoparticles against Aedes aegypti larvae. Parasitol Res 110:175-184

Soni N, S Prakash (2012b). Synthesis of gold nanoparticles by the fungus Aspergillus niger and its efficacy against mosquito larvae. Rep Parasitol 2012; Article 2 
Subramaniam J, K Kovendan, PM Kumar, K Murugan, W Walton (2012). Mosquito larvicidal activity of aloe vera (family: Liliaceae) leaf extract and Bacillus sphaericus, against chikungunya vector, Aedes aegypti. Saud J Biol Sci 19:503-509

Sundaravadivelan C, MN Padmanabhan (2014). Effect of mycosynthesized silver nanoparticles from filtrate of Trichoderma harzianum against larvae and pupa of dengue vector Aedes aegypti 1. Environ Sci Pollut Res 21:4624-4633

Tang S, J Zheng (2018). Antibacterial activity of silver nanoparticles: structural effects. Adv Healthc Mater 7; Article e1701503

Teixeira IF, EC Barbosa, SCE Tsang, PH Camargo (2018). Carbon nitrides and metal nanoparticles: From controlled synthesis to design principles for improved photocatalysis. Chem Soc Rev 47:7783-7817

Thompson JD, DG Higgins, TJ Gibson (1994). Clustal W: Improving the sensitivity of progressive multiple sequence alignment through sequence weighting, position-specific gap penalties and weight matrix choice. Nucl Acid Res 22:4673-4680

Tyagi S, PK Tyagi, D Gola, N Chauhan, RK Bharti (2019). Extracellular synthesis of silver nanoparticles using entomopathogenic fungus: Characterization and antibacterial potential. SN Appl Sci 1; Article 1545

Vahabi K, GA Mansoori, S Karimi (2011). Biosynthesis of silver nanoparticles by fungus Trichoderma reesei (a route for large-scale production of agnps). Insci J 1:65-79

Vivekanandhan P, S Deepa, EJ Kweka, MS Shivakumar (2018a). Toxicity of Fusarium oxysporum-vkfo-01 derived silver nanoparticles as potential inseciticide against three mosquito vector species (Diptera: Culicidae). J Clust Sci 29:1139-1149
Vivekanandhan P, T Kavitha, S Karthi, S Senthil-Nathan, MS Shivakumar (2018b). Toxicity of Beauveria bassiana-28 mycelial extracts on larvae of Culex quinquefasciatus mosquito (Diptera: Culicidae). Intl J Environ Res Publ Health 15; Article 440

Wahid F, XJ Zhao, SR Jia, H Bai, C Zhong (2020). Nanocomposite hydrogels as multifunctional systems for biomedical applications: Current state and perspectives. Compos B Eng 200; Article 108208

Wang Y, N Herron (1991). Nanometer-sized semiconductor clusters: Materials synthesis, quantum size effects and photophysical properties. J Phys Chem 95:525-532

WHO (2018). Guidelines For Laboratory And Field Testing of Mosquito Larvicides. Geneva: World Health Organization; 2005 (Document Who/Cds/Whopes/Gcdpp/13.[Google Scholar])

WHO (1981). Instructions for Determining the Susceptibility or Resistance of Mosquito Larvae to Insecticides. World Health Organization, Rome italy

Yadav SK (2010). Pesticide applications-threat to ecosystems. J Hum Ecol 32:37-45

Zhang D, XL Ma, Y Gu, H Huang, GW Zhang (2020). Green synthesis of metallic nanoparticles and their potential applications to treat cancer. Front Chemis 8; Article 799

Zhao B, J Yan, S Zhang, X Liu, Z Gao (2014). Phylogeny and pathogenicity of Fusarium spp. isolated from greenhouse melon soil in liaoning province. Saud J Biol Sci 21:374-379

Zhao G, SE Stevens (1998). Multiple parameters for the comprehensive evaluation of the susceptibility of Escherichia coli to the silver ion. Biometals 11:27-32 